\title{
MedienPädagogik
}

Zeitschrift für Theorie und Praxis der Medienbildung

Jahrbuch Medienpädagogik 2.

Zweitveröffentlichung aus: Jahrbuch Medienpädagogik 2. (2001) Opladen: Leske + Budrich. Herausgegeben von Ben Bachmair, Dieter Spanhel und Claudia de Witt.

\section{Die Virtuelle Universität. Idee, Konzepte, Erfahrungen}

\author{
Horst Dichanz
}

Daß ein Student aus Kolumbien mit einem Kommilitonen aus Japan in der Cafeteria sitzt und sich über eine soeben gehörte Vorlesung unterhält, das könnte an jeder Universität passieren. Daß der eine Informatiker, der andere Geisteswissenschaftler ist, obwohl beide am gleichen Seminar teilgenommen haben, dürfte schon seltener sein. Und daß sich die beiden während ihrer Unterhaltung überhaupt nicht sehen, ist ein sicheres Indiz dafür, daß sie gemeinsam an einer virtuellen Universität (http://vu.FernUni-hagen.de/ feuvus_f.html) studieren.

An der FernUniversität ist das zwar nicht täglich der Fall, aber doch keine Ausnahme mehr. „Wir versuchen, mit der Virtuellen Universität den Anforderungen an künftige Bildungssysteme gerecht zu werden“, formuliert Gunter Schlageter, Professor für Praktische Informatik, das Ziel des in seiner Größe bislang einmaligen Telelearning-Projekts in der Bundesrepublik: „Wir reagieren auf die immer rascher sich entwickelnden Berufsprofile und die dynamischen Arbeitsstrukturen, indem wir uns neuer Informations- und Kommunikationssysteme bedienen“, sagt Schlageter. Schließlich seien Berufsleben und Bildung in Zukunft nicht mehr wie bisher voneinander zu trennen. „Mit Hilfe der neuen Medien läßt sich Online-Coaching ebenso realisieren wie ein self-placed learning“, erklärt der Universitätslehrer (NET investor, 1/99).

\section{Die Idee}

Die FernUniversität Hagen ist eine wissenschaftliche Hochschule des Landes NordrheinWestfalen. Sie ist die einzige staatliche FernUniversität in Deutschland, an der 1999 56000 Studierende immatrikuliert waren. Die Studenten kommen aus allen Bundesländern, 7,3\% studieren aus dem benachbarten Ausland.

Wer an der FernUni in Hagen studiert, braucht nicht mehr in engen Hörsälen um einen Sitzplatz zu kämpfen. Ein Computer und ein Internet-Browser zu Hause reichen völlig aus. Der Dozent oder seine Vorlesung erscheinen unter 
http://www.FernUni-hagen.de auf dem Bildschirm. Über die Homepage der FernUniversität können zahlreiche Serviceangebote und Studienhilfen erreicht werden. Seit dem Wintersemester 1996/97 ist die Uni über den Bildschirm zu erreichen. Bis zum Jahr 2000 sollen ein großer Teil der Lehr- und Weiterbildungsangebote virtualisiert sein. Mit ihrem umfassenden Konzept des virtuellen Studiums nimmt die FernUni Hagen eine Vorreiterrolle in Europa ein.

Die virtuelle Universität ist nicht mehr um Hörsäle herumgebaut; vielmehr bietet sie den Studierenden ihre Studienmöglichkeiten in größtmöglicher räumlicher und zeitlicher Unabhängigkeit an. Zum überwiegenden Teil arbeiten die Studierenden an sehr unterschiedlichen Orten, zu Hause, auf Reisen, im Büro, auch in der Uni. Der Personal Computer spielt eine entscheidende Rolle: der Rechner ist zugleich Auskunftsterminal und Kommunikationszentrum, Anbieter von Lehrmaterial, Experimentierumgebung, Bibliothek. Zum Lehrmaterial gehören Multimedia-Kurse, (interaktive) Videos, Computer Based Training, Simulationspakete, Experimentiersoftware, Animationen, Printmedien (auch Ausdruck entsprechend dem aktuellen Bedarf).

Das Projekt „Virtuelle Universität“ geht über bisherige Ansätze multimedialen Studierens hinaus, da sie alle Funktionen einer Universität zu einem vollständigen System virtuell integriert. Hierzu gehört nicht nur interaktives Lehrmaterial, das über Netz verschickt wird (auf diesen Aspekt beschränken sich viele Ansätze zur „Online-Universität“) - vielmehr sind für einen erfolgversprechenden Ansatz unabdingbar: umfassende Kommunikationsmöglichkeiten, insbesondere auch für gemeinsames Lernen (peer-learning) zwischen den Studierenden und für die soziale Vernetzung, Möglichkeiten zur Gruppenund Seminararbeit über Netz, neue Formen des Übungs- und Praktikumsbetriebs über Netz, bequemer Zugang zur (traditionellen und digitalen) Bibliothek und zur Administration, Informierungs- und Beratungssysteme. Das System beruht auf üblichen Personal Computern, nutzt insbesondere Internet und ISDN, es schließt netzbasiertes Videoconferencing ein.

An der Virtuellen Universität wird großer Wert darauf gelegt, daß den Studenten all die Bereiche virtuell offen stehen, die eine Hochschule üblicherweise anbietet. Lehre, Betreuung, Verwaltung, Bibliothek und Studentensekretariat sind Bestandteile des alltäglichen virtuellen Lebens. Damit der soziale Kontakt nicht abreißt, gibt es auch eine Virtuelle Cafeteria und ein Schwarzes Brett, um all die täglichen Dinge zu erledigen und zu besprechen, die zum Studentenleben gehören. Multimediale Kurse sind ebenso im Angebot wie interaktive Videos, Computer based Training, Simulationspakete, Experimentiersoftware, Animationen oder auch Printmedien wie Bücher oder Fachzeitschriften, die online recherchiert und bestellt werden können. Die virtuelle Betreuung durch den Dozenten ist gesichert. Statt langer Wartezeiten besteht die Möglichkeit, Anfragen per Email zu stellen. Im Jahr 1999 nutzten bereits 6000 Studenten die virtuellen Studienangebote.

Es ist schon selbstverständlich, daß der Kontakt zu den Kommilitonen, aber auch zu den Tutoren oder den Lehrenden über das Internet entweder auf schriftlichem, akustischem oder gar visuellem Weg hergestellt wird, je nach 
Ausrüstung und Möglichkeiten der Gesprächsteilnehmer. Noch wird nicht ganz darauf verzichtet, daß der Student - wenn auch selten - seine Uni persönlich aufsucht, z.B. für Prüfungen.

\section{Aus der Praxis der Virtuellen Universität (VU)}

Die beiden Standbeine der VU sind die Lehr- und Forschungsarbeiten der einzelnen Lehrstühle und die technisch-fernstudiendidaktische Kompetenz des Zentrums für Fernstudienentwicklung (ZFE). Dieses universitäre Medienzentrum entwickelt in Kooperation mit den Lehrstühlen der Fachbereiche der FernUniversität Bausteine für die Lehre der „Virtuellen Universität“. Zu diesen Bausteinen zählen: ${ }^{1}$

- Multimediale Lehrsoftware,

- Multimediale Dateikurse,

- Dateikurse im Internet,

- Video und Fernsehen „on demand“,

- Diskussionsgruppen und virtuelle Seminare,

- Simultankonferenzen (Text, Ton, Video),

- Interaktive Übungen und Aufgaben.

Zusätzlich bieten die Lehrstühle in Eigenregie folgende online-Dienste an:

- Beratung und Betreuung persönlich und per Telefon und

- Lehrgebietsinformationen in Broschüren und im Internet.

Auf diese Weise wird ein dichtes Netz von Information und Beratung angeboten, das eine optimale Betreuung der Studierenden sicherstellt. Die einzelnen Bausteine übernehmen folgende Funktionen:

\section{Multimediale Lehrsoftware}

Dieser Baustein der virtuellen Universität zeichnet sich durch hohe Interaktivität aus und ist besonders gut geeignet zum tätigen Erschließen, Einüben und Vertiefen wissenschaftlicher Sachverhalte (Modellsimulationen, Laborsimulationen). Damit umfangreiche verbale Informationen, die die Wissenschaft letzten Endes immer begleiten, vermittelt werden können, hat das ZFE eine „sprachbegleitete Lehrsoftware“ entwickelt. Diese vermittelt verbale Informationen im wesentlichen über die Tonspur, während der Bildschirm frei bleibt für Bilder, Animationen und Prozesse, die von Stichwörtern, Hinweisen und Kommentaren begleitet werden. Einzelne Tonsequenzen können beliebig oft wiederholt werden.

1 Der folgende Text enthält z.T. Textpassagen aus der Broschüre „Bausteine der ,Virtuellen Universität““ (ZFE, Hagen 1997), die als „offizielle Texte“ der FeU nicht alle im Detail gekennzeichnet sind. 


\section{Multimediale Dateikurse}

Diese textbegleitete Lehrsoftware vermittelt in der Tradition des Studienbriefes die notwendigen verbalen Informationen mit Hilfe des geschriebenen Textes. Ein Großteil der klassischen Kurse der FernUni liegt bereits in digitaler Form vor und kann daher gut zu multimedialen Dateikursen weiterentwickelt werden. Multimedial ist der Dateikurs insofern, daß er ergänzend zum geschriebenen Text weitere mediale Elemente integriert und so das alte didaktische Prinzip des Medienverbundes fortführt und auf eine höhere Stufe hebt. Zu den integrierbaren medialen Elementen eines Dateikurses zählen insbesondere Abbildungen aller Art, Animationen, Ton- und Videosequenzen sowie Simulationen. Multimediale Lehrmaterialien werden zur Zeit auf CD-ROM verbreitet. Sie können aber auch jederzeit über Netze verteilt werden, wenn diese ausreichend leistungsfähig und kostengünstig sind.

\section{Dateikurse im Internet}

Die Dateikurse werden schon jetzt im Internet verbreitet. Sie sind nicht nur textgleich zu den gedruckten Versionen, sondern auch layoutgleich. Das ist vorteilhaft, solange es elektronische und gedruckte Versionen nebeneinander gibt. Die Dateikurse im Internet nutzen nicht nur die Interaktivität, sondern auch alle Übergänge in das WWW. So wurden z.B. alle Pflichtkurse des Grundstudiums Erziehungswissenschaft (47 Kurseinheiten $=33$ SWS) in Dateikurse umgearbeitet.

Video und Fernsehen „on demand“

Neben Videosequenzen zur Integration in multimediale Lehrmedien gibt es selbständige Video- und Fernsehprogramme als Bausteine der virtuellen Universität. Die Video- oder Fernsehfassung der Programme sind im Inhalt identisch, sie unterscheiden sich bisher noch in der Verbreitungsform. Doch auch dieser Unterschied wird verschwinden, wenn beide in Zukunft „online“ und „on demand“ abrufbar sind. Zusätzlich werden beide mit weiteren Informationen und Rückkanälen ergänzt. Die Fernsehreihe „FernUniversität im Dritten“, die in Zusammenarbeit mit dem WDR produziert und im WDR ausgestrahlt wird, ist jederzeit im Internet abrufbar. Rund ein Dutzend Videotitel der FernUniversität sind versuchsweise im Infocity-Netz der Vebacom zugänglich gemacht.

\section{Diskussionsgruppen und virtuelle Seminare}

Von Anfang an sah die FernUni Hagen die Kommunikation mit den Studenten als sehr wichtige Aufgabe des Lehrens und Lernens aus der Ferne. Heute sind durch die neuen Kommunikationstechniken die Voraussetzungen dafür 
besser denn je. Zu den nicht-simultanen Kommunikationsmitteln zählen Diskussionsgruppen und virtuelle Seminare. Die virtuellen Seminare haben inzwischen einen gewissen Standard und Alltagsroutine erreicht. Sie werden bei den Studenten durchweg sehr positiv beurteilt. Laut einer Befragung des Instituts für Psychologie schätzen die Teilnehmer die neuen Kommunikationsmöglichkeiten, die den Kontakt und die Kommunikation mit der FernUniversität erleichtern, die Zusammenarbeit in Arbeitsgruppen ermöglichen und zu produktiven Kontakten führen, „die weit über das jeweilige virtuelle Seminar hinaus bestehen bleiben“ (Rogalla, 1998, S. 34). Die Feststellung der Psychologen gilt auch für andere Bereiche der Universität: „Die virtuellen Seminare ... haben das Experimentierstadium verlassen“ (ebd.). Die virtuellen Seminare werden von den einzelnen Lehrgebieten durchgeführt und betreut. Diskussionsgruppen gibt es zu technischen Fragen (von URZ) und zu allgemeinen sowie speziellen inhaltlichen Themen. Die Diskussionsgruppen sind für jedermann von überall her zugänglich.

\section{Simultankonferenzen (Text, Ton, Video)}

Simultankonferenzen können auf Text beschränkt, aber auch um Ton und Video ergänzt sein. Sie sind jedoch für Zwecke der Lehre und des Studiums nicht ganz so hilfreich wie die nicht-simultane Kommunikation. Als viel geeigneter erweisen sich dagegen tonund videogestützte Konferenzen, die den Bildschirm von Texten weitgehend frei halten. Tongestützte Konferenzen eignen sich besonders gut zur Besprechung von Haus- oder Magisterarbeiten oder zum Gedankenaustausch über Entwicklungs- und Forschungsprojekte. Typische Anwendungsfelder von Videokonferenzen sind an der FernUni mündliche Prüfungen (besonders im Fachbereich ESGW) und Fernseminare, auch mit sehr großen Teilnehmerzahlen (z.B. Wirtschaftswissenschaften). Eine besondere Form der Simultankonferenzen sind die seit ca. 3 Jahren eingeführten Video-Prüfungen. Bei ihnen werden mit Hilfe der Videotechnik Prüfungen von Kandidaten im Ausland (z.B. Österreich, Schweiz) durchgeführt. Sie ersparen den Studierenden die aufwendigen Reisen nach Hagen. Bisher wurden schon über 100 Video-Prüfungen durchgeführt.

\section{Interaktive Übungen und Aufgaben}

$\mathrm{Zu}$ den klassischen Veranstaltungen universitärer Lehre zählt die Übung (zur Vorlesung). Sie wird an der FernUni ersetzt oder ergänzt durch Einsende- und Selbstkontrollaufgaben, die schon seit vielen Jahren zu einem großen Teil maschinell auswertbar sind. Die Online-Version des interaktiven Übungs- und Aufgabentypus unter Nutzung elektronischer Netze wird zu einem wichtigen kommunikativen Baustein der virtuellen Universität. Bisher kostet die Belegung von online- und CD-ROM-Kursen genauso viel wie ihre Papierversion (18,- DM). Da eine CD-ROM oder ein Internetkurs wesentlich 
billiger herzustellen sind, wird sich diesbezüglich noch einiges ändern. Das wird auch im Interesse der Studierenden sein, da auf sie bei der Belegung eines Internetkurses zusätzliche Kosten wie Telefongebühren für die Internetverbindung, evtl. Providergebühren sowie Druckkosten zukommen.

\section{Kommunikation an der virtuellen Universität}

Zur Kommunikation dienen im Angebot der Uni Hagen neben Chat-Foren und Email auch Voice-Mail sowie Tele- und Videokonferenzen. In der Ausbildung setzt die Hochschule neben Multimedia-Kursen, Videos, Simulationspaketen und CD-ROMs immer noch Printmedien ein. Im „Shop“ können Studenten zusätzlich Materialien zu Lehrveranstaltungen oder zur Weiterbildung durchstöbern, abrufen oder gegen eine Gebühr bestellen. Das kostet Geld. Doch nahezu alle Fernstudenten sind berufstätig und können diese Kosten eher aufbringen als ihre Kommilitonen an der klassischen Universität mit Hörsälen und Mensa.

\section{Studium im Gefängnis - Chancen an der FernUni Hagen}

Wer im Gefängnis studieren will, kann das beispielsweise an der FernUni Hagen tun. Dort sind seit dem ersten Studienjahr 1974 Inhaftierte eingeschrieben. Seit 1983 konnte die FernUni außerdem in einem Studienzentrum an der JVA Geldern ausgiebig Erfahrungen mit inhaftierten Studenten sammeln (vgl. Campus \& Karriere, 24.02.1999).

\section{Die Virtuelle Universität im Internet - Die Benutzeroberfläche}

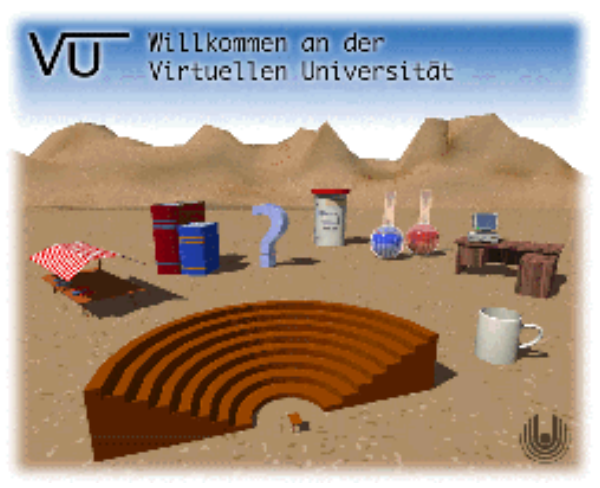

Elemente der Benutzeroberfläche sind:

Lehre: Zugang zu den Lehrveranstaltungen (Vorlesungen, Prüfungen usw.), Online-Seminare, Online-Übungsgruppen oder die Durchführung von Prakti- 
ka, Unterstützung von Teamarbeit der Studierenden mittels geeigneter Softwaresysteme (CSCW).

Büro: Zugang zu den administrativen Funktionen (Belegung von Kursen, Rückmeldung, Änderung persönlicher Daten usw.)

Information: Zentrale Anlaufstelle für jegliche Fragen. Die Information enthält ein Informationssystem und verweist auf entsprechende Informationsseiten. Bei Bedarf wird zu menschlichen Beratern durchgestellt. Über „guided tours“ werden am Fernstudium interessierte Besucher über den virtuellen Universitätscampus geführt.

News: Universitätsweites schwarzes Brett mit aktuellen Informationen. Strukturierung und Suchfunktionen unterstützen die Studierenden bei der Auswahl der relevanten Informationen.

Forschung: Präsentation von Forschungsschwerpunkten, Veröffentlichung und Diskussion von Forschungsergebnissen einzelner Fachgebiete.

Colloquien und Chats: Diese Funktion ist nicht nur wichtig für die Forschenden der Universität, sondern auch für Diplomanden, Doktoranden, Projektgruppen usw.

Cafeteria: Forum für soziale Kontakte unter den Studierenden. Mit Hilfe verschiedener Kommunikationswerkzeuge können hier Fragen zum Studium oder Dinge des täglichen Lebens diskutiert werden. Zusätzlich werden schwarze Bretter angeboten, an denen Studierende Aushänge anbringen können.

Bibliothek: Bequemer Zugang zu traditionellen und digitalen Bibliotheken zur Durchführung von Recherchen, Vormerkung und Bestellung von Büchern etc. Über die Bibliothek können auch in digitaler Form verfügbare Bücher oder Artikel eingesehen und auf das lokale System übertragen werden.

Shop: Präsentation von Lehr- und Weiterbildungsmaterial, das gegen Gebühr abgegeben wird. Dieses Angebot ist von speziellem Interesse für das „Training on the job“, da man jederzeit auf das benötigte Material aus der jeweiligen beruflichen Situation heraus zugreifen kann.

\section{Zur weiteren Entwicklung}

Wichtige künftige Entwicklungsbereiche im Rahmen der Virtuellen Universität umfassen die Weiterentwicklung der technischen Plattform (Datenschutz, Integration von Verwaltungsabläufen etc.) und die Weiterentwicklung von Lehrformen wie virtuelle Seminare, Online-Übungen und Praktika unter Einbeziehung der bisher gewonnenen Erfahrungen.

Die technische Plattform ist offen für verschiedene didaktische Konzepte und Materialien unterschiedlichster Wissensbereiche. Sie stützt sich auf das Internet und integriert Datenbanken und Java-Technologien. Auf der Benut- 
zerseite basiert sie auf Standardsoftware. Dadurch ist es leicht möglich, das System auf andere technische Plattformen aufzusetzen und auf vielfältige Bereiche der Aus- und Weiterbildung (Universitäten, Fachhochschulen, Schulen, firmeninterne Weiterbildung etc.) zu übertragen.

Die Virtuelle Universität eröffnet völlig neue Perspektiven hinsichtlich der Qualität, der Individualisierung, der Bedarfsorientierung und der sozialen Vernetzung des Studiums. Sie muß unter neuen Gesichtspunkten eines technologisch gestützten Medienverbundes fortgesetzt und erweitert werden. Unter den Einflüssen der IT verändern sich auch die Möglichkeiten und Strukturen des Fernstudiums und damit der Aufgaben der FernUniversität. Die niederländische FernUniversität („Open Universiteit“) bekommt dies bereits zu spüren: Dort ändert sich bereits der Kontakt zwischen Lehrenden und Lernenden im Fernstudium, so daß auch über veränderte Aufgaben der FernUniversität nachgedacht wird.

\section{Bildungspolitische Perspektiven der FernUniversität}

Die bisherigen Erfahrungen und Forschungsergebnisse aus einzelnen Projekten weisen darauf hin, daß die bisher praktizierte mediale Präsentation von Informationen für Lernzwecke immer weniger ausreicht. Sie muß um lernstrategische Hilfen und Übungen ergänzt werden. Deshalb wäre eine Virtuelle Universität schlecht beraten, wenn sie sich technisch und konzeptionell als eigene, autarke, quasi isolierte Bildungsinstitution begreift und sich als geschlossenes System etabliert. In Verbindung mit den neuen technischen Möglichkeiten verdient die Entwicklung von Netzwerken besondere Beachtung. Erfahrungen und Beobachtungen aus dem Ausland zeigen, daß wir einen deutlichen Rückstand aufzuholen haben. Auch die Kommission für Studienreform im Land NRW sieht die Notwendigkeit, daß nicht nur die Hochschulen, sondern Bildungsinstitutionen aller Art auf lokaler Ebene mit anderen Institutionen, z.B. mit der Arbeitsverwaltung in Netzen Zusammenarbeiten. Solche „Angebote im Kooperationsverbund tragen ... wesentlich dazu bei, Kommunikationsbarrieren gruppen-, fach- und hochschulübergreifend zu überwinden und damit die ,Singularität der Lebensverhältnisse’ zu relativieren“ (Kommission für Studienreform 1996, S. 54).

Bedingt durch die technisch leicht zu realisierenden Individualisierungen von Programm- und Lehrangeboten haben große, durchstrukturierte Gesamtangebote und Medienverbundsysteme wie z.B. die traditionellen Funk- oder Telekollegs in der Konkurrenz zu anderen, individuell flexibel zu nutzenden Medienangeboten an Attraktivität verloren. Die FernUniversität als virtuelle Universität hat gerade erst begonnen, mit den zahlreichen hieraus sich ergebenden Konsequenzen für Aufgaben, Organisationsformen und Kooperationen Erfahrungen zu sammeln. Der damit verbundene Reformbedarf ist noch nicht vollständig erkannt und beschrieben. Er wird aber auch die Arbeit der Präsenzhochschulen mit betreffen. 
FernUniversität, Medienuniversität, Virtuelle Universität

Im Rahmen der Weiterentwicklung der FernUniversität zu einer Medienuniversität, die die Virtuelle Universität einschließen sollte, sind einige Entwicklungen zu beachten, die auch in der FernUniversität diskutiert werden: Die technische Weiterentwicklung von Mediensystemen und die (medien-) politisch gewollte Erweiterung der Angebote der öffentlichen und privaten Programmanbieter (z.B. der Rundfunkanstalten) durch Online-Support-Systeme zeigen zahlreiche neue Übergänge bisher getrennter Systeme und Dienste, die auch die traditionelle Organisation des Fernstudiums betreffen und verändern. Indizien im In- und Ausland deuten darauf hin, daß die Zukunft von Kommunikations- und Wissenssystemen in der Entwicklung kombinierbarer und kombinierter Netzwerke liegt, in denen Online-Zugriffe und Internet nur zwei von vielen anderen Funktionen sind. Bei der z.Z. stattfindenden Neustrukturierung nationaler und zunehmend auch internationaler Bildungsnetzwerke wird eine neue Positionsbestimmung des Fernstudiums und damit der FernUniversität erforderlich. Bereits heute beginnen Präsenzhochschulen, mit Femstudienelementen zu experimentieren (vgl. Verbund Südwest, Hochschulnetzwerk der Bertelsmann Stiftung). Die FernUniversität sollte ein Interesse daran haben, an diesen Entwicklungen teilzunehmen und sich mit ihren Aufgaben und Lösungsideen zu plazieren.

Ein wichtiger und auch für die Entwicklungen der Virtuellen Universität notwendiger Sektor sind die internationalen Online-Dienste und deren Ordnung und Bearbeitung auf regionaler, nationaler und internationaler (europäischer) Ebene durch Server. Hier sind zumindest für die BRD die wesentlichen Entscheidungen gefallen und die Strukturen vorgezeichnet. Immerhin sollte der FernUniversität an einer überzeugenden Präsentation in all diesen Diensten gelegen sein. Im Landesserver ist die FernUniversität mit ihrer differenzierten Homepage gut vertreten, im Deutschen Bildungsserver mit zwei Spezialzugriffen, die aber nicht zur Homepage führen, in übernationalen Servern (ARIADNE-Projekt, GEM, Eurydice-Website, European SchoolNet (EUN) (vgl. GIB-News 1/98) ist die Präsenz der FernUniversität schwach. Diese Server sollten möglichst umgehend bedient und die Präsentation der FernUniversität dort sorgfältig gepflegt werden.

Eine Studie der Bertelsmann- und der Nixdorf-Stiftung prognostiziert für das Jahr 2005, daß mehr als die Hälfte aller Studierenden in einer Distance-Leaming-Organisation immatrikuliert sein werden; aber auch dieses Studium wird nicht nur Online erfolgen. Vielmehr werden neben den Wissensangeboten Informations- und Navigationssysteme eine immer größere Rolle spielen. Die derzeitigen Veränderungen der bildungsrelevanten Rundfunk- und Fernsehprogramme vermitteln schon heute einen Eindruck von der wachsenden Integration der verschiedenen Dienste in Verbindung mit den öffentlichen Medien (vgl. BR, SWR, Pro Sieben mit seinem Magazin Othello). Die FernUniversität könnte und sollte die Chance nutzen, die sich durch den augenblicklichen Stillstand bei der Entwicklung eines europäischen Bildungskanals im Rahmen der 
EBU abzeichnet, um europa-orientierte Initiativen zu planen und sich auf diese Weise als eine europäische Femstudieninstitution in Erinnerung zu bringen. Von besonderer Bedeutung dürfte die Entwicklung von Konzepten und Strukturen sein, die in einer Art „neuem Medienverbund“ die verschiedenen Elemente von Bildungsangeboten unter Einbeziehung aller Programmanbieter und unter Nutzung aller technischen Möglichkeiten (vgl. Lemaire 1999) die Bildungslandschaft informatorisch übersichtlich ordnen und Navigationshilfen fiir die Abnehmer/Interessenten entwickeln. Ihre derzeit starke Position in der EADTU könnte ihr hierfür geeignete Kanäle öffnen.

Als Folge der Öffnung medialer Angebote und in Verbindung mit den o.g. strukturellen Entwicklungen kann die FernUniversität eine Weiterentwicklung ins Auge fassen, für die sich folgende Sektoren anbieten:

- Internationale Erweiterung/Ergänzung vorhandener Fachangebote

- Errichtung internationaler Studiengänge mit international anerkannten

- Abschlüssen

- Bewerbungen bei D\&R-Projekten der EU

- Förderung des Austausches mit anderen (europäischen) FernUniversitäten

- Fortsetzung des Engagements bei der Durchführung übernationaler Tagungen u.ä.

- Herausgabe von/Beteiligung an einer internationalen Online-Zeitschrift für Distance Education.

Die hier skizzierte Entwicklung der FernUniversität zielt darauf, ihre bisherigen Leistungen und Erfahrungen - vor allem auf dem Sektor der Mediennutzung - auszuwerten, den bisherigen computerbasierten Ansatz der virtuellen Universität deutlich zu erweitern, die Möglichkeiten der FernUniversität bei der künftigen Medienentwicklung in Deutschland und in Europa zur Geltung zu bringen und sie zu einer modernen Medienuniversität weiterzuentwickeln.

\section{Literatur}

Bausteine der „Virtuellen Universität“, Broschüre des Zentrums für Femstudienentwicklung, FernUniversität Hagen 1997 Campus \& Karriere, Ausgabe vom 24. 02. 1999

Encarnacao, J. 'LVLeidhold, W./Reuter, A.: Hochschulentwicklung durch neue Medien, Vision 2005. Hrsg. v. d. Bertelsmann Stiftung/Heinz Nixdorf Stiftung Kommission fiir Studienreform NRW: 1996, S. 54

Lemaire, L: Die Offene Universität der Informationsgesellschaft, in: Grundlagen der Weiterbildung 1/1999, S. 31-33

Ministerium für Bildung, Kultur und Wissenschaft: OcenW, Oct. 1999, Nr. 06D

NET investor. 1/99

Rogalla, I.: Virtuelle Methodenseminare im Institut für Psychologie. Evaluationsbericht.

Berichte aus dem Arbeitsbereich Psychologie. FernUniversität Hagen 1998, Nr. 28

Petrovic, O./Kailer, N./Scheff, J.: Lernformen, Einsatzdefizite und -potentiale in Unternehmen und Universitäten. In: GdWZ 10 (1999) 2, S. 62-64

ZFE-Broschüre: Bausteine der „Virtuellen Universität“. Hagen 1997 